\title{
An Iterative Method for Extreme Optics of Two-Level Systems
}

\author{
R. Parzyński*, M. Sobczak and A. Plucińska \\ Faculty of Physics, Adam Mickiewicz University \\ Umultowska 85, 61-614 Poznań, Poland
}

(Received August 12, 2004; in final form November 5, 2004)

\begin{abstract}
We formulate the problem of a two-level system in a linearly polarized laser field in terms of a nonlinear Riccati-type differential equation and solve the equation analytically in time intervals much shorter than half the optical period. The analytical solutions for subsequent intervals are then stuck together in an iterative procedure to cover the whole scale time of the laser pulse. Very good quality of the iterative method is shown by recovering with it a number of subtle effects met in earlier numerically calculated photon-emission spectra from model molecular ions, double quantum wells, atoms, and semiconductors. The method is used to describe novel, carrier-envelope offset phase effects in the region of extreme nonlinear optics, i.e., when two-level systems are exposed to pulses of only a few cycles in duration and strength ensuring the Rabi frequency to approach the laser light frequence.
\end{abstract}

PACS numbers: 42.50.Md, 42.50.Hz, 42.65.Ky, 42.65.Re

\section{Introduction}

In the theory of light-matter interactions there is probably no more fundamental model than the two-level one [1]. Over the last decade, for example, the model has succeeded in explaining the main features of propagation of strong few-cycle pulses through atomic and semiconductor media [2-12], e.g., carrier wave Rabi flopping, third-harmonic generation in disguise of second harmonic, and carrier-envelope phase effects, to name a few. It has also made a basis for the description of high-order harmonic generation from a single atom [13], a symmetric molecular ion [14-16] and a double-well quantum structure [17-19] with emphasis

*corresponding author; e-mail: parzynsk@amu.edu.pl 
on the strongly non-perturbative picture of the phenomenon and the occurrence of peaks in the spectrum of coherently scattered light at the positions of even harmonics. When applied to double wells, the model turned out to be successful also in explaining the effect of laser control of tunneling [20].

Despite its dissemination in atomic, molecular, and solid state physics the two-level model of light-matter interaction still suffers from the lack of exact analytical solution covering the whole range of laser intensities as well as pulse shapes and durations. The analytical solutions known hitherto cover only some different limiting cases. For instance, the most celebrated rotating-wave-approximation (RWA) solution [1] is restricted to laser intensities ensuring the resonance Rabi frequency, $\Omega_{\mathrm{R}}$, to be much smaller than the laser frequency, $\omega$. Beyond RWA, the known analytical solutions include the non-RWA corrections along a perturbative procedure (e.g. [21, 22]), either are valid in the so-called multiphoton excitation region $[14,15]\left(\omega \ll \omega_{21}\right.$ along with $\Omega_{\mathrm{R}} \ll \omega_{21}$, where $\omega_{21}$ stands for the frequency separation between the two levels) or in the quite opposite strong coupling region [14-19] $\left(\omega \gg \omega_{21}\right.$ and $\left.\Omega_{\mathrm{R}} \gg \omega_{21}\right)$. Probably, the only analytical solution covering the whole intensity region is the recent one of Tritschler et al. [23] for a box-shaped pulse, but obtained within the so-called square-wave approximation (SWA) consisting in replacing the actual time behavior of the field within half the optical cycle by a square of a constant appropriately chosen magnitude. Being approximate, this solution was able to reproduce only qualitatively some features of the exact numerical calculations, especially for the case of the resonant excitation $\left(\omega=\omega_{21}\right)$, but was less convincing for distinctly off-resonant excitation.

The aim of our paper is to present a quick iterative procedure for the problem of the two-level system in linearly polarized laser field, based on an analytical solution of the Schrödinger equation in very short time intervals. The analytical solution turned out to be possible thanks to defining the problem of level populations in terms of a single nonlinear Riccati-type differential equation in conjunction with dividing each halfcycle of the pulse into a number of narrow slices of equal width and considering constant the electric field within each slice with a value determined by the pulse function at the middle of the slice. The analytically obtained solutions for all slices in the pulse are then stick together by a simple recurrence formula relating the boundary conditions in the adjacent slices. For each slice, this approach offers a simple analytical formula for the ratio of level population amplitudes, resulting in equally simple analytical formulae for population inversion, induced dipole moment, and spectrum of the radiation emitted by this dipole. A good quality of the method is proved by showing that the resulting photon-emission spectra reproduce the numerically calculated ones available in literature [8, 9, 13-19]. Moreover, the method indicates weak points of the square-wave solution of Tritschler et al. [23] and is shown to be particularly useful in the area of extreme nonlinear optics [23], i.e., when few-cycle, strong pulses stimulate significant population dynamics in a two-level system on a timescale of half the 
optical cycle. In particular we apply the iterative method to describe the recently observed effect of pulse carrier-envelope offset phase on harmonic generation.

\section{The analytical solution for short time intervals and iterative method}

When presenting our analytical solution for short time intervals we start with the standard expansion $\psi(t)=b_{1}(t)|1\rangle+b_{2}(t)|2\rangle$ for the wave function of the two-level system in a laser field, where $|1\rangle$ and $|2\rangle$ stands for the time-independent opposite-parity eigenstates of the bare system with eigenfrequencies $\omega_{1}$ and $\omega_{2}$, respectively. The above choice of $\psi(t)$ with a complete neglect of continuous states is justified only when the regime of strong coupling between the two bound states (the Rabi frequency comparable to the laser light frequency) is achievable at laser intensities not producing significant ionization. The systems to be considered, with their large bound-bound transition dipole moments (molecular ions at large internuclear distances, quantum wells, semiconductors), fall into this regime already at less than $10 \mathrm{TW} / \mathrm{cm}^{2}$ intensities at which ionization can be disregarded to the first approximation. The time-dependent population amplitudes of the levels, $b_{1}(t)$ and $b_{2}(t)$, are then governed [16] by the equation

$$
\mathrm{i} \frac{\mathrm{d}}{\mathrm{d} t} b_{k}=\omega_{k} b_{k}-\Omega(t) b_{l}(t)
$$

where both $k$ and $l$ run the values 1,2 with the constraint $l \neq k$, and $\Omega(t)=\Omega_{\mathrm{R}} h(t)$ is the instantaneous Rabi frequency with $\Omega_{\mathrm{R}}=\mu \epsilon_{0} / \hbar$ being the usual Rabi frequency as determined by the dipole transition matrix element $\mu=\langle 1|e z| 2\rangle$ and the electric field amplitude $\epsilon_{0}$, while $h(t)=f(t) \sin (\omega t+\phi)$ describes the incident-field evolution with $f(t)$ having the sense of pulse shape (for pulses of at least few cycles in duration), $\omega$ the carrier frequency and $\phi$ the carrier-envelope offset phase. The latter is known $[8,9,12,24-27]$ to be a relevant quantity determining the response of the system in the regime of few-cycle pulses.

Traditionally, there has been solved in different coupling regimes either a set of two linear differential equations for $b_{k}$ with no RWA applied (e.g. [14-17]) or more often (e.g. [13-15, 19, 23]) the resulting set of three linear differential equations for the Bloch vector components: $u=2 \operatorname{Re}\left(b_{1}^{*} b_{2}\right), v=2 \operatorname{Im}\left(b_{1}^{*} b_{2}\right), w=$ $\left|b_{2}\right|^{2}-\left|b_{1}\right|^{2}$. Instead, we prefer to work with only one but nonlinear differential equation for the ratio $r(t)=b_{2}(t) / b_{1}(t)$ of the population amplitudes. Through the population conservation law, $\left|b_{1}\right|^{2}+\left|b_{2}\right|^{2}=1$, the above $r$ determines directly both the population inversion $w=\left(|r|^{2}-1\right) /\left(|r|^{2}+1\right)$ and the induced dipole moment $d(t)=\langle\psi(t)|e z| \psi(t)\rangle=\mu u=2 \mu \operatorname{Re}(r) /\left(|r|^{2}+1\right)$ and, consequently, the spectrum of coherently scattered light as well. After differentiating $r$ over time and then using Eq. (1) one obtains [21, 22] $r$ to fulfil the following differential equation: 


$$
\mathrm{i} \frac{\mathrm{d} r}{\mathrm{~d} t}=\left(r^{2}-1\right) \Omega(t)+\omega_{21} r
$$

where $\omega_{21}=\omega_{2}-\omega_{1}$ is the frequency separation between the bare levels. This equation falls into the family of nonlinear Riccati-type equations and a way for its iterative solution results from the transformation

$$
\begin{aligned}
& r(t)=\frac{1}{2 \Omega(t)}\left[\Omega^{\mathrm{eff}}(t) R(t)-\omega_{21}\right], \\
& \Omega^{\mathrm{eff}}(t)=\sqrt{4 \Omega^{2}(t)+\omega_{21}^{2}},
\end{aligned}
$$

converting Eq. (2) into

$$
\mathrm{i} \frac{\mathrm{d} R}{\mathrm{~d} t}=\left(R^{2}-1\right) \frac{\Omega^{\text {eff }}}{2}+\mathrm{i}\left(\frac{\omega_{21}}{\Omega^{\text {eff }}} R-1\right) \frac{\omega_{21}}{\Omega^{\text {eff }} \Omega} \frac{\mathrm{d} \Omega}{\mathrm{d} t} .
$$

To avoid the cumbersome second term on the right-hand side, including the derivative $\mathrm{d} \Omega / \mathrm{d} t$, we divide the timescale of the pulse into a number of sufficiently narrow intervals with $t_{j}^{\mathrm{i}} \leq t_{j} \leq t_{j}^{\mathrm{f}}$ being the running time within the $j$-th interval. In each interval of its width much shorter than half an optical cycle we approximate the Rabi frequencies $\Omega\left(t_{j}\right)$ and $\Omega^{\text {eff }}\left(t_{j}\right)$ as constants of the values which they actually take in the middle $\left(t_{j}^{\mathrm{m}}\right)$ of the interval. Under such an approximation, Eq. (5), when adapted to the $j$-th interval looks like $\mathrm{i}\left(\mathrm{d} R_{j} / \mathrm{d} t_{j}\right)=\left(R_{j}^{2}-1\right) \Omega_{j}^{\text {eff }} / 2$, where $\Omega_{j}^{\mathrm{eff}}=\Omega^{\mathrm{eff}}\left(t_{j}=t_{j}^{\mathrm{m}}\right)$. The resulting equation has straightforward analytical solution

$$
R_{j}\left(t_{j}\right)=\frac{1-\mathrm{i} R_{j}^{\mathrm{in}} \cot \left[\Omega_{j}^{\mathrm{eff}}\left(t_{j}-t_{j}^{\mathrm{i}}\right) / 2\right]}{R_{j}^{\mathrm{in}}-\mathrm{i} \cot \left[\Omega_{j}^{\mathrm{eff}}\left(t_{j}-t_{j}^{\mathrm{i}}\right) / 2\right]},
$$

where $R_{j}^{\text {in }}=R_{j}\left(t_{j}=t_{j}^{\mathrm{i}}\right)$ is the initial value of $R_{j}$, i.e., that at the beginning of the $j$-th interval. For the extreme time in the interval, $t_{j}=t_{j}^{\mathrm{f}}$, we have $R_{j}\left(t_{j}=t_{j}^{\mathrm{f}}\right)=R_{j+1}^{\mathrm{in}}$, resulting in the recurrence formula for the initial conditions

$$
R_{j+1}^{\mathrm{in}}=\frac{1-\mathrm{i} R_{j}^{\mathrm{in}} \cot \left[\Omega_{j}^{\mathrm{eff}}\left(t_{j}^{\mathrm{f}}-t_{j}^{\mathrm{i}}\right) / 2\right]}{R_{j}^{\mathrm{in}}-\mathrm{i} \cot \left[\Omega_{j}^{\mathrm{eff}}\left(t_{j}^{\mathrm{f}}-t_{j}^{\mathrm{i}}\right) / 2\right]} .
$$

As a consequence of Eqs. (6) and (7) we obtain from Eq. (3) the solution for $r_{j}\left(t_{j}\right)$ :

$$
r_{j}\left(t_{j}\right)=\frac{2 \Omega_{j}-\left\{\omega_{21}+\mathrm{i} \Omega_{j}^{\mathrm{eff}} \cot \left[\Omega_{j}^{\mathrm{eff}}\left(t_{j}-t_{j}^{\mathrm{i}}\right) / 2\right]\right\} r_{j}^{\mathrm{in}}}{\omega_{21}-\mathrm{i} \Omega_{j}^{\mathrm{eff}} \cot \left[\Omega_{j}^{\mathrm{eff}}\left(t_{j}-t_{j}^{\mathrm{i}}\right) / 2\right]+2 \Omega_{j} r_{j}^{\mathrm{in}}}
$$

and also the recurrence formula for the initial conditions, $r_{j}^{\text {in }}$, at the beginnings of subsequent time intervals

$$
r_{j+1}^{\mathrm{in}}=\frac{2 \Omega_{j}-\left\{\omega_{21}+\mathrm{i} \Omega_{j}^{\mathrm{eff}} \cot \left[\Omega_{j}^{\mathrm{eff}}\left(t_{j}^{\mathrm{f}}-t_{j}^{\mathrm{i}}\right) / 2\right]\right\} r_{j}^{\mathrm{in}}}{\omega_{21}-\mathrm{i} \Omega_{j}^{\mathrm{eff}} \cot \left[\Omega_{j}^{\mathrm{eff}}\left(t_{j}^{\mathrm{f}}-t_{j}^{\mathrm{i}}\right) / 2\right]+2 \Omega_{j} r_{j}^{\mathrm{in}}},
$$

where $\Omega_{j}^{\text {eff }}=\sqrt{4 \Omega_{j}^{2}+\omega_{21}^{2}}$ with $\Omega_{j}=\Omega_{\mathrm{R}} h_{j}$ and $h_{j}=f\left(t_{j}^{\mathrm{m}}\right) \sin \left(\omega t_{j}^{\mathrm{m}}+\phi\right)$. The 
solutions in the form of Eqs. (8) and (9) allow us to obtain population inversion, induced dipole moment, and electric field of coherently scattered light within the subsequent time intervals, $t_{j}^{\mathrm{i}} \leq t_{j} \leq t_{j}^{\mathrm{f}}$, and to stick the solutions for the intervals to cover the whole timescale of the incident pulse.

Before writing down the final formulae it is convenient to introduce the dimensionless strength parameter $x=\Omega_{\mathrm{R}} / \omega$, the dimensionless level separation parameter $y=\omega_{21} / \omega$ and the dimensionless time parameter $\tau=\omega t$, where $0 \leq$ $\tau \leq 2 \pi N$ for a $N$-cycle pulse. Then, we divide each halfcycle in the $\tau$ domain into $K$ intervals of width $\pi / K$ each, letting $j$ to fall into the range $1 \leq j \leq 2 N K$. Within the $j$-th interval, the running time covers the range $(j-1) \frac{\pi}{K}=\tau_{j}^{\mathrm{i}} \leq \tau_{j} \leq$ $\tau_{j}^{\mathrm{f}}=j \frac{\pi}{K}$ and the middle of the interval occurs at $\tau_{j}^{\mathrm{m}}=j \frac{\pi}{K}-\frac{\pi}{2 K}$. Also, we make the replacement $r_{j}^{\text {in }}=I_{j}$ and introduce the normalized effective Rabi frequency within the $j$-th interval as $x_{j}^{\text {eff }}=\Omega_{j}^{\text {eff }} / \omega=\sqrt{4 x_{j}^{2}+y^{2}}$, where $x_{j}=x h_{j}$ with $h_{j}=f\left(\tau_{j}^{\mathrm{m}}\right) \sin \left(\tau_{j}^{\mathrm{m}}+\phi\right)$. In this language the recurrence formula of Eq. (9) reads

$$
I_{j+1}=\frac{2 x_{j}-\left[y+\mathrm{i} x_{j}^{\mathrm{eff}} \cot \left(\pi x_{j}^{\mathrm{eff}} / 2 K\right)\right] I_{j}}{y-\mathrm{i} x_{j}^{\mathrm{eff}} \cot \left(\pi x_{j}^{\mathrm{eff}} / 2 K\right)+2 x_{j} I_{j}} .
$$

For a given field-system parameters $x, y, \phi, f(\tau)$, Eq. (10) allows us to generate the initial conditions for all subsequent $2 N K$ time intervals from the only known initial condition $I_{1}$ for the first interval ( $I_{1}=0$ throughout this paper). Having generated the initial conditions we calculate the evolution of population inversion within the $j$-th time interval from

$$
\begin{gathered}
w_{j}\left(\tau_{j}\right)=\frac{-1}{\left(1+\left|I_{j}\right|^{2}\right)\left(x_{j}^{\mathrm{eff}}\right)^{2}}\left\{y\left[y\left(1-\left|I_{j}\right|^{2}\right)+4 x_{j} \operatorname{Re}\left(I_{j}\right)\right]\right. \\
+4 x_{j}\left[x_{j}\left(1-\left|I_{j}\right|^{2}\right)-y \operatorname{Re}\left(I_{j}\right)\right] \cos \left[x_{j}^{\mathrm{eff}}\left(\tau_{j}-\tau_{j}^{\mathrm{i}}\right)\right] \\
\left.-4 x_{j} x_{j}^{\mathrm{eff}} \operatorname{Im}\left(I_{j}\right) \sin \left[x_{j}^{\mathrm{eff}}\left(\tau_{j}-\tau_{j}^{\mathrm{i}}\right)\right]\right\}
\end{gathered}
$$

while the evolution of the induced dipole moment from

$$
\begin{aligned}
& d_{j}\left(\tau_{j}\right)=\frac{2 \mu}{\left(1+\left|I_{j}\right|^{2}\right)\left(x_{j}^{\mathrm{eff}}\right)^{2}} \\
& \quad \times\left\{x_{j}\left[y\left(1-\left|I_{j}\right|^{2}\right)+4 x_{j} \operatorname{Re}\left(I_{j}\right)\right]-y\left[x_{j}\left(1-\left|I_{j}\right|^{2}\right)-y \operatorname{Re}\left(I_{j}\right)\right]\right. \\
& \left.\quad \times \cos \left[x_{j}^{\mathrm{eff}}\left(\tau_{j}-\tau_{j}^{\mathrm{i}}\right)\right]+y x_{j}^{\mathrm{eff}} \operatorname{Im}\left(I_{j}\right) \sin \left[x_{j}^{\mathrm{eff}}\left(\tau_{j}-\tau_{j}^{\mathrm{i}}\right)\right]\right\}
\end{aligned}
$$

where $0 \leq \tau_{j}-\tau_{j}^{\mathrm{i}} \leq \pi / K$ within each interval. Taking the second derivative of Eq. (12) with respect to $\tau_{j}$ results in the electric field of the coherently scattered light in the forward direction 


$$
\begin{aligned}
\epsilon_{j}\left(\tau_{j}\right) & \sim \frac{2 \mu y}{1+\left|I_{j}\right|^{2}} \\
& \times\left\{\left[x_{j}\left(1-\left|I_{j}\right|^{2}\right)-y \operatorname{Re}\left(I_{j}\right)\right] \cos \left[x_{j}^{\mathrm{eff}}\left(\tau_{j}-\tau_{j}^{\mathrm{i}}\right)\right]\right. \\
& \left.-x_{j}^{\mathrm{eff}} \operatorname{Im}\left(I_{j}\right) \sin \left[x_{j}^{\mathrm{eff}}\left(\tau_{j}-\tau_{j}^{\mathrm{i}}\right)\right]\right\} .
\end{aligned}
$$

To study spectra we need to take Fourier transforms $\left(\tau_{j} \rightarrow z\right.$, where $z$ is the spectrometer frequency in units of the incident light frequency $\omega$ ) of Eqs. (12) and (13) with the results

$$
\begin{aligned}
& d_{j}(z)=\frac{\mu \mathrm{e}^{-\mathrm{i} z(j-1) \pi / K}}{\left(1+\left|I_{j}\right|^{2}\right)\left(x_{j}^{\mathrm{eff}}\right)^{2}}\left\{\mathrm { i } \left[x_{j}\left[y\left(1-\left|I_{j}\right|^{2}\right)+4 x_{j} \operatorname{Re}\left(I_{j}\right)\right] 2 f_{j}^{0}\right.\right. \\
& \left.\left.-y\left[x_{j}\left(1-\left|I_{j}\right|^{2}\right)-y \operatorname{Re}\left(I_{j}\right)\right]\left(f_{j}^{-1}+f_{j}^{+1}\right)\right]+y x_{j}^{\mathrm{eff}} \operatorname{Im}\left(I_{j}\right)\left(f_{j}^{-1}-f_{j}^{+1}\right)\right\}
\end{aligned}
$$

and

$$
\begin{aligned}
& \epsilon_{j}(z) \sim \frac{\mu y \mathrm{e}^{-\mathrm{i} z(j-1) \pi / K}}{1+\left|I_{j}\right|^{2}} \\
& \times\left\{\mathrm{i}\left[x_{j}\left(1-\left|I_{j}\right|^{2}\right)-y \operatorname{Re}\left(I_{j}\right)\right]\left(f_{j}^{-1}+f_{j}^{+1}\right)-x_{j}^{\mathrm{eff}} \operatorname{Im}\left(I_{j}\right)\left(f_{j}^{-1}-f_{j}^{+1}\right)\right\},
\end{aligned}
$$

where

$$
f_{j}^{q}=\frac{\mathrm{e}^{-\mathrm{i}\left(z+q x_{j}^{\text {eff }}\right) \pi / K}-1}{z+q x_{j}^{\text {eff }}}
$$

with $q=0, \pm 1$. Finally, to cover the whole timescale of the pulse one needs to sum up Eqs. (11)-(15) over $j$, taking into account Eqs. (10) and (16).

\section{Quality of the iterative method}

We have extensively examined the accuracy of the iterative method (Eqs. (10)-(16)) in wide ranges of pulse shapes $f(\tau)$, pulse strengths $x$, carrier frequencies $y$, and carrier-envelope phases $\phi$. In any case the method was found to be able to fit the results of direct numerical integrations of the Riccati-type Eq. (2), provided that $K$, i.e., the number of intervals into which we divide each optical halfcycle was chosen appropriately. Generally, the higher $K$ the better was the quality of the method, as expected. However, $K$ of the order of only a few units or at most ten appeared to be sufficient to ensure good-quality of the method for not too strong pulses $(x \leq 1)$. For extremely strong pulses $(x \gg 1)$, generating fast population dynamics on a timescale of an optical cycle, an increase in $K$ was needed for the method to reproduce all details of the numerical solution. However, even in the latter case only a little of computer time was consumed to accomplish successfully the iterative procedure. 
To exemplify the effect of better quality of the iterative method when increasing $K$, let us focus on the one-photon resonance $(y=1)$ by a pulse of moderate strength $(x=1)$. We intentionally take this case because it is covered neither by the strong-coupling ( $y \ll 1$ and $x \gg y$ ) analytical solution of Ivanov et al. [16, 17] nor by the multiphoton-excitation $(y \gg 1$ and $x \ll y)$ analytical solution of Zuo et al. [14, 15]. Moreover, to assess the SWA solution of Tritschler et al. [23] we choose a box-shaped $(f(\tau)=1)$ sine-like $(\phi=0)$ pulse. The SWA, originally applied to the system of optical Bloch equations, consisted in replacing the sequence of halfcycles of the electric field by the sequence of identical squares, each of a height ensuring the areas under the halfcycle and square to be equal. In terms of our short-time-interval analytical solution, SWA corresponds to the choice of $K=1\left(h_{j}=(-1)^{j+1}\right)$ and to rescaling $x \rightarrow \frac{2}{\pi} x$ resulting in $x_{j}=(-1)^{j+1} \frac{2}{\pi} x$. In this limit our equations for $w_{j}\left(\tau_{j}\right)$ and $d_{j}\left(\tau_{j}\right)$ convert into those of Tritschler et al. obtained by a different analytical approach exploiting the Bloch equations. For the pulse of $N=2$ cycles in duration, now available in the laboratory practice (e.g. [23]), we show in Fig. 1a the iteratively calculated effect of $K$ on the population inversion (solid lines), and compare this result with that obtained by
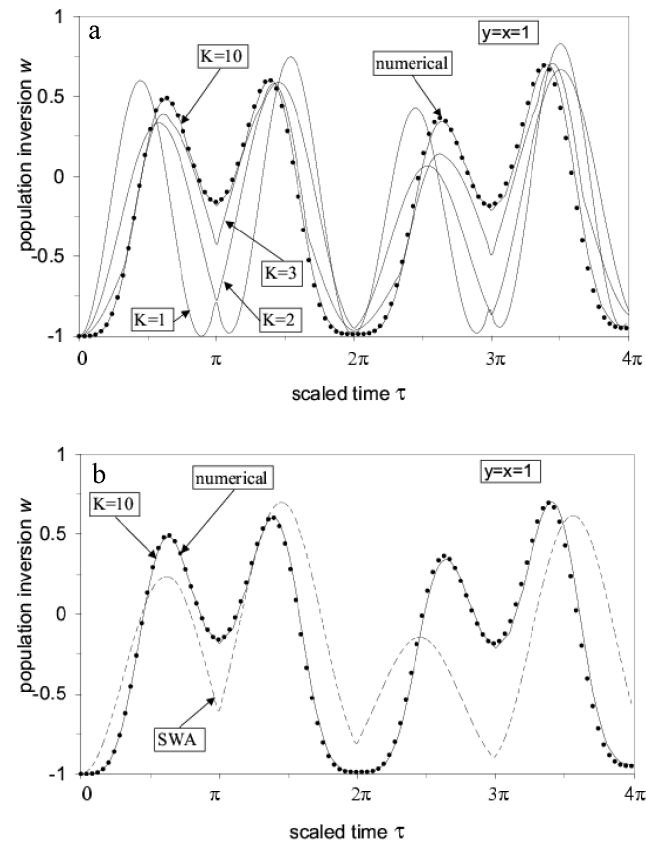

Fig. 1a, b. The evolution of population inversion $w$ versus $K$ under the box-shaped $(f(\tau)=1)$ sine-like $(\phi=0)$ two-cycle $(N=2)$ pulse of $x=y=1$. Solid lines the results of the iterative procedure exploiting Eq. (11), dotted lines - the results of direct numerical integration of the Riccati-type Eq. (2) for $r$, dashed line - the results of square wave approximation. 
integrating numerically the Riccati-type Eq. (2) for $r$ (dotted line). As seen, the choice of $K=10$ ensures nearly perfect coincidence between the two approaches. On the other hand, Fig. 1b provides a comparison between our iterative results at $K=10$ and the SWA results (dashed line) leading to a conclusion that the square-wave approximation can be used only to general predictions of qualitative nature.

To prove a good quality of our iterative method we now recover with it some numerically calculated spectra of light coherently scattered by two-level systems, available in the literature. One such a two-level system that has received a lot of attention in the past is the lowest pair of different-symmetry electronic levels of the $H_{2}^{+}$molecular ion $\left(1 \sigma_{\mathrm{g}}\right.$ and $\left.1 \sigma_{\mathrm{u}}\right)$, a pair being well isolated from other levels particularly at large internuclear distances. In particular, Zuo et al. show in Fig. 6b of their paper [15] the two-level numerically calculated photon-emission spectrum of $\mathrm{H}_{2}^{+}$in the near-resonance region translating into our $y=1.1$ and $x=1.86$. The spectrum was obtained by assuming the $f(\tau) \cos (\tau)$ electric field with $f(\tau)$ Gaussian increasing by 10 optical cycles $\left(f(\tau)=\exp \left\{-[(\tau-20 \pi) / 10 \pi]^{2}\right\}\right.$ for $0 \leq \tau \leq 20 \pi)$ and then keeping a constant value up to 30 cycles $(f(\tau)=1$ for $20 \pi<\tau \leq 60 \pi$ ). For the above conditions, we apply our Eq. (14) (with $\mu$ put to 1) along with Eq. (10) to present in Fig. 2a the iteratively calculated spectrum $|d(z)|^{2}=\left|\sum_{j} d_{j}(z)\right|^{2}$ with $1 \leq j \leq 2 N K=60 K$. To achieve high resolution of our spectrum we chose $K=100$ and we will maintain this choice through all other figures to be presented. Our spectrum of Fig. 2a consists of the Mollow triplets occurring at each odd-order harmonic $(1,3,5$, and 7$)$ with the same side band separation within the triplets. This iteratively obtained structure is in full agreement with the numerical spectrum of Zuo et al. (Fig. 6b in [15]). In a different paper Zuo et al. [14] give, for the $f(\tau) \cos \tau=\cos \tau$ field, their numerical spectrum for the same system but under the so-called strong-coupling conditions meaning in our notation $y=0.445$ and $x=1.9\left(x / y=\Omega_{\mathrm{R}} / \omega_{21}=4.27\right)$. Under these conditions our iterative spectrum generated from Eq. (14) for $N=30$ cycle pulse is shown in Fig. 2b. An interesting feature of the iterative spectrum are (besides the familiar odd-order harmonics 3, 5, and 7) the doublets around the positions of even harmonics caused by the large Rabi splittings of the odd harmonics. This spectrum is a counterpart of the numerical spectrum of Zuo et al. (Fig. 7 in [14]).

Also Ivanov et al. [16, 17] have calculated the emission spectra from molecular ions but using their analytical formula (Eq. (52) in [16]) derived in the limiting case of extremely strong coupling ( $y \ll 1$ and $x \gg y$ in our language). We applied our Eq. (14) to this region and obtained with it the results shown in Fig. 3. This figure presents the heights of the odd-harmonic peaks, $H(n)$, normalized to the third harmonic peak, for $y=0.1$ and two values of $x=14.5$ and 15 , respectively. We have assumed a 30-cycle pulse of the form $f(\tau) \cos \tau$ with $f(\tau)=1$. Our Fig. 3, obtained along the iterative procedure, coincides perfectly with the appropriate results of Ivanov and Corkum (Fig. 3 in [16]). 

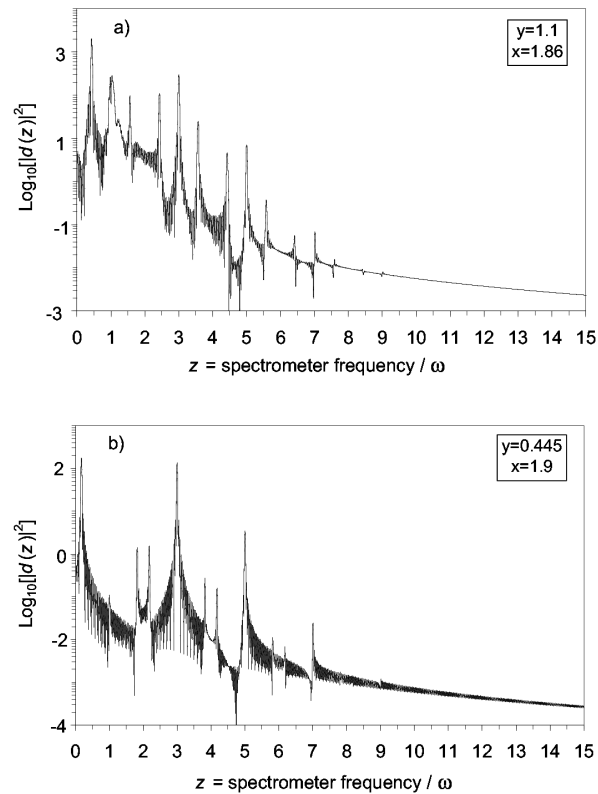

Fig. 2. Photon-emission spectra, $|d(z)|^{2}$, from a model two-level molecular hydrogen ion, calculated iteratively by using Eq. (14). (a) Near-resonance case $(y=1.1$ and $x=1.86$ ) for the $f(\tau) \cos (\tau)$ pulse with $f(\tau)$ Gaussian increasing by 10 optical cycles and then keeping the value of 1 up to 30 cycles; (b) strong-coupling case ( $y=0.445$ and $x=1.9$ ) for the 30 -cycle $f(\tau) \cos (\tau)$ pulse with $f(\tau)=1$. The conditions are as those in the papers by Zuo et al. (Fig. 6b in [15] and Fig. 7 in [14], respectively). For the high-resolution of the presented spectra we choose $K=100$.

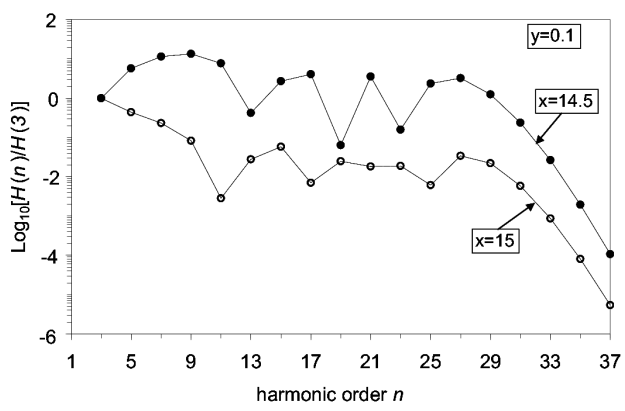

Fig. 3. Normalized heights, $H(n)$, of the odd-harmonic peaks calculated iteratively with the use of Eq. (14), for the case of extremely strong coupling $(y \ll 1, x \gg y)$ of the two-level system to 30-cycle pulse of $f(\tau) \cos (\tau)$ form with $f(\tau)=1$. The conditions are as those in the paper by Ivanov and Corkum (Fig. 3 in [16]).

A different place where two-level approximation has appeared to be reliable is a symmetric double quantum well [17-20] extensively studied in the context of 
laser control of tunneling and symmetry breaking with strong short pulses. The latter effect results in the appearance of spectral peaks at the positions of even harmonics from the systems with inversion symmetry. For example, Levinson et al. (Fig. 2 in their paper [19]) give the spectra from the double-well structure obtained by integrating numerically the set of three Bloch equations for the $f(\tau) \cos \tau$ pulse with $f(\tau)=1$. The frequency-strength parameters in their numerical calculations fall into the strong-coupling region $(y=0.625, x=1.25$ in one case (their Fig. 2a) and $y=0.589, x=1.178$ in the other case (their Fig. 2b)). For the above two sets of frequency-strength parameters we show in Fig. 4 our iterative spectra resulting from Eq. (14) for the 30-cycle pulse assumed. The asymmetric doublets at the positions of the second and fourth harmonics, formed when using the first set of parameters (Fig. 4a), are seen to coalesce into single peaks when taking the other set (Fig. 4b). Moreover, the second set of parameters results in shifting the low-frequency component of the spectrum towards zero. Both behaviors of our iteratively obtained spectra are the same as those in the numerical spectra of Levinson et al. (Fig. 2 in [19]) and are connected with approaching the so-called accidental degeneracy of two Floquet states of the system [17, 18, 20] at some parameters. The parameter $x$ from the second set does nearly satisfy
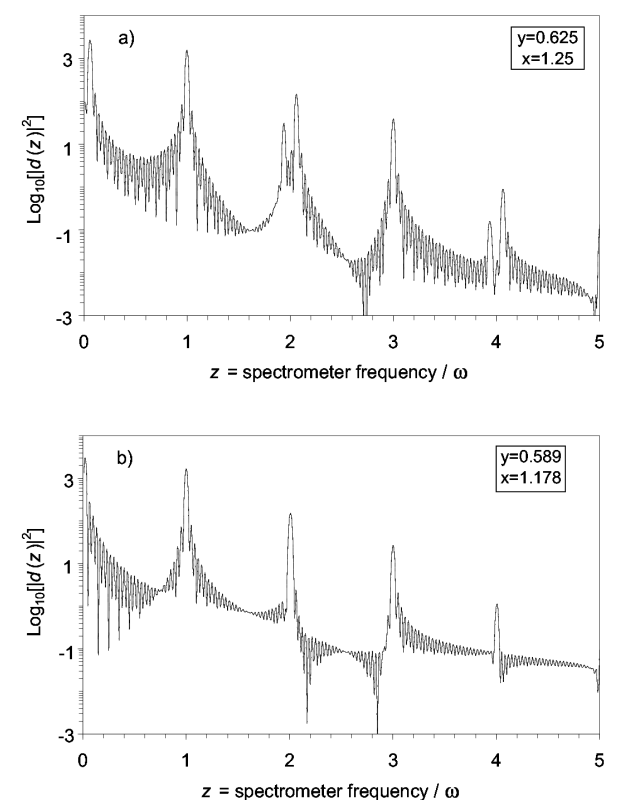

Fig. 4. Spectra from a symmetric double-well structure obtained iteratively by applying Eq. (14), for 30-cycle pulse of $f(\tau) \cos (\tau)$ type with $f(\tau)=1$ and frequency-strength parameters $y=0.625, x=1.25$ (a) and $y=0.589, x=1.178(\mathrm{~b})$. The conditions are as those in the paper by Levinson et al. (Fig. 2 in [19]). 
the condition of the accidental degeneracy, i.e., it ensures for the Bessel function $J_{0}(2 x)$ to drop to zero $[17,20]$.

Mücke et al. [8] have also used the two-level model to simulate numerically the spectra of light emitted around the third harmonic from GaAs semiconductor exposed to 5 fs pulse of $\operatorname{sech}\left(\tau / \tau_{0}\right) \cos \tau$ form, where $\tau_{0}=\tau_{\text {FWHM }} / 1.763$. The results of their simulations (Fig. 2 in [8]) reveal the evolution of the third-harmonic peak into a doublet structure when increasing the envelope pulse area $A$. For the $\operatorname{sech}\left(\tau / \tau_{0}\right)$ envelope, the area $A$ is related to our $x$ parameter through $A=\pi \tau_{0} x=$ $\left(2 \pi^{2} / 1.763\right) N_{\text {FWHM }} x$, where $N_{\text {FWHM }}$ is the full width at half maximum (FWHM) measured in optical cycles $\left(N_{\mathrm{FWHM}}=1.71\right.$ in this case $)$. For the conditions close to those of Mücke et al., we present in Fig. 5 our iterative spectra, obtained from Eq. (15). Our spectra are a qualitative reproduction of the numerical spectra of Mücke et al. (Fig. 2 in [8]). A possible source of only qualitative agreement in this case is that our spectra are the pure response of the system, i.e., with
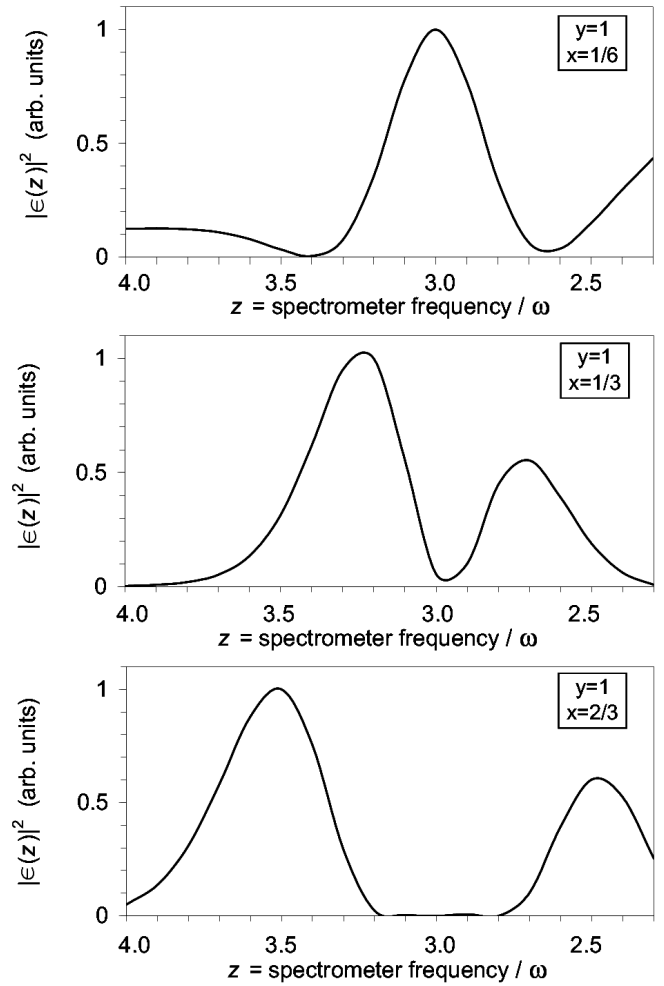

Fig. 5. Evolution of the third-harmonic peak into a doublet with increasing pulse strength parameter $x$, calculated iteratively with the use of Eq. (15). The conditions ( 5 fs pulse of $\operatorname{sech}\left(\tau / \tau_{0}\right) \cos (\tau)$ form) are close to those of numerical simulations by Mücke et al. (Fig. 2 in [8]). The positions of peaks in the doublet agree with those from numerical simulations but the heights are reversed. 
no propagation effects included which were naturally taken into account in the simulations of Mücke et al. by coupling the Bloch equations to the Maxwell equations.

\section{Carrier-envelope phase effects}

We now apply the iterative method to calculate, for a particular case, the dependence of the two-level-system response on the phase difference $(\phi)$ between carrier wave and the maximum of the pulse envelope. To be specific, we make recurrence to the carrier-wave Rabi flopping originally studied numerically by Hughes [4] for a resonant $(y=1)$ pulse $h(\tau)=\operatorname{sech}\left(\tau / \tau_{0}\right) \sin (\tau)$ of a fixed FWHM $\left(N_{\mathrm{FWHM}}=1.72\right)$ but different pulse envelopes $A=19.24 x$. By coupling the optical Bloch equations to the Maxwell equations, Hughes considered propagation of the $A=2 l \pi$ pulses through a two-level medium, where $l$ was an integer. For the areas $A=6 \pi-14 \pi$ of Hughes, the left column of Fig. 6 shows population differences, $\left|b_{1}\right|^{2}-\left|b_{2}\right|^{2}$, versus time obtained by our iterative procedure with the use of Eq. (11). Our results practically do not differ from the original numerical results obtained by Hughes just near the front-face of the two-level material, i.e., where the propagation effects were not important yet (the left column of Fig. 3 in [4]). Our graphs confirm the original result of Hughes on incomplete Rabi flops at $A \geq 8 \pi$. On the other hand, the right column of our Fig. 6 shows our iteratively obtained population differences but for the $h(\tau)=\operatorname{sech}\left(\tau / \tau_{0}\right) \cos (\tau)$ pulse, i.e., the pulse with its carrier-envelope phase $\phi$ shifted by $\pi / 2$ with respect to the pulse used by Hughes. Some differences caused by this shift are clearly seen in the middle parts of the population difference curves. These parts correspond to the times for which the pulse intensity has already evolved to its high values. The main differences introduced by changing the carrier-envelope phase $\phi$ consist in either converting the double peaks into single ones (and vice versa) or inverting the asymmetry in double peaks.

The above $\phi$-sensitivity of population inversion produces the dependence of the spectrum of scattered light on carrier-envelope phase. In Fig. 7a, we show the spectrum calculated iteratively with the use of Eq. (15) for the Hughes pulse $h(\tau)=\operatorname{sech}\left(\tau / \tau_{0}\right) \sin (\tau+\phi)$, i.e., with $\phi=0, N_{\text {FWHM }}=1.72, y=1$ and $x=1.31$ (this $x$ corresponds to the envelope pulse area $A=8 \pi$ ). Except the spectral peak at the fundamental frequency $(z=1)$, one sees a well pronounced peak at the position of second harmonic $(z=2)$ because the chosen $x$ is in the vicinity of the value (1.178) ensuring the accidental degeneracy $\left(J_{0}(2 x)=0\right)$ of the Floquet states of the system (compare Fig. 4 and its discussion). A similar peak around $z=2$ was found by Tritschler et al. (Fig. 1a in [12]) on the basis of their numerical solution of the two-level Bloch equations for a different pulse envelope, namely $\operatorname{sinc}\left(\tau / \tau_{0}\right)=\sin \left(\tau / \tau_{0}\right) /\left(\tau / \tau_{0}\right)$ instead of $\operatorname{sech}\left(\tau / \tau_{0}\right)$, and different light-matter parameters $\left(N_{\text {FWHM }}=1.81, y=2, x=0.76\right)$. In addition to Fig. 7 a, we show 

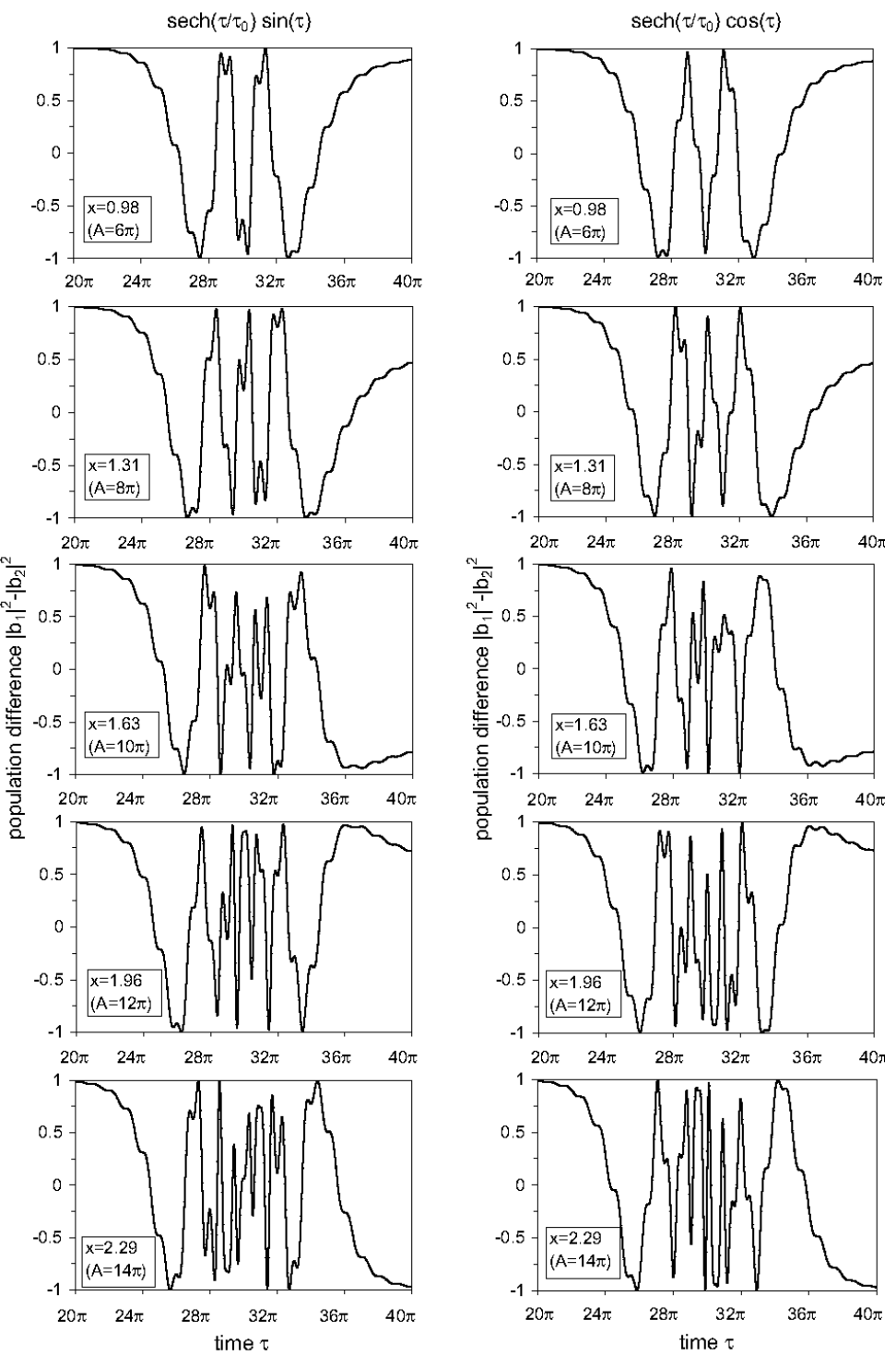

Fig. 6. Population difference, $\left|b_{1}\right|^{2}-\left|b_{2}\right|^{2}$, between the ground and excited states versus time, for $h(\tau)=\operatorname{sech}\left(\tau / \tau_{0}\right) \sin (\tau)$ pulse (left column) and $h(\tau)=\operatorname{sech}\left(\tau / \tau_{0}\right) \cos (\tau)$ pulse (right column), i.e., the pulses with their carrier-envelope phases shifted to each other by $\pi / 2$. The graphs were obtained iteratively by using Eq. (11). The chosen parameters: $N_{\mathrm{FWHM}}=1.72, y=1$, while the envelope pulse areas $A$ and the corresponding strength parameters $x$ are shown in the graphs. The left graphs reproduce the fully numerical results of Hughes (Fig. 3 in [4]). 

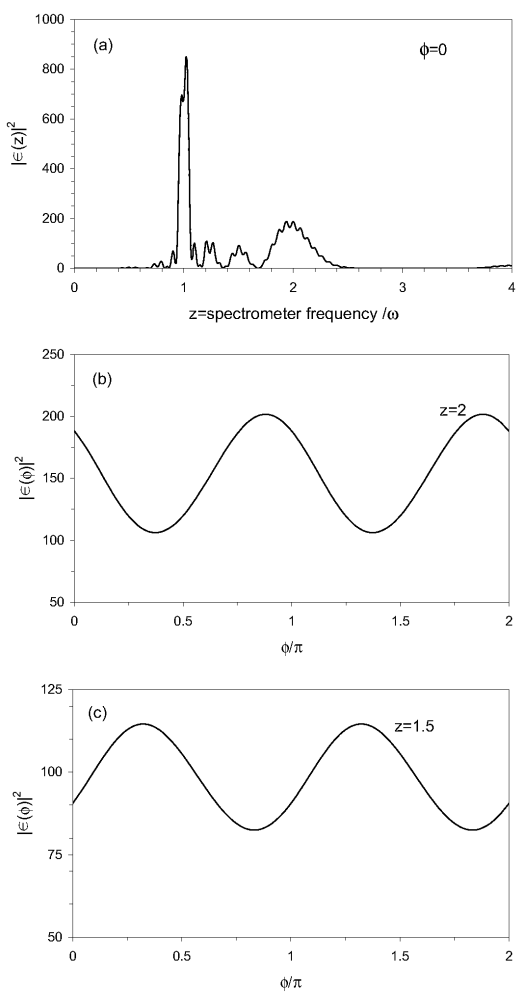

Fig. 7. (a) The iteratively calculated (from Eq. (15)) photon-emission spectrum generated by $h(\tau)=\operatorname{sech}\left(\tau / \tau_{0}\right) \sin (\tau+\phi)$ pulse of the parameters $\phi=0, N_{\mathrm{FWHM}}=1.72$, $y=1$ and $x=1.31$ ( $8 \pi$ pulse). (b) The calculated (from Eq. (15)) height of the spectral peak at the position of second harmonic $(z=2)$ versus carrier-envelope offset phase $\phi$. The $\phi$-dependence with a period of $\pi$ agrees with the results of fully numerical calculations by other authors $[9,12]$ for different pulse shapes and light-matter parameters. (c) The same as (b) but for the small peak at $z=1.5$ in (a).

in Fig. 7b the dependence (calculated iteratively from Eq. (15)) of the height of the spectral peak at $z=2$ on the carrier-envelope phase $0 \leq \phi \leq 2 \pi$ in the pulse $h(\tau)=\operatorname{sech}\left(\tau / \tau_{0}\right) \sin (\tau+\phi)$. The $\phi$-dependence is well seen and has a period of $\pi$ in agreement with fully numerical calculations of Tritschler et al. [12] and Mücke et al. (Fig. 1b in [9]) exploiting the optical Bloch equations. The same periodicity is seen in Fig. 7c corresponding to the small peak in Fig. 7a around $z=1.5$.

\section{A simpler version of the iterative method}

We have checked that the results shown in Figs. 1-7 are also obtainable from a simpler version of Eqs. (10)-(13) consuming less of computer time. This version is obtained by, first, replacing $\tau_{j}$ in Eqs. (11)-(13) by its extreme value 
$\tau_{j} \rightarrow \tau_{j}^{\mathrm{f}}=j \pi / K$ and, then, choosing $K$ sufficiently large for the argument of the inherent trigonometric functions to be a small quantity $\left(\pi x_{j}^{\text {eff }} / K \ll 1\right)$. In this limit, the trigonometric functions are replaced by their leading terms $\left(\cos \left(\pi x_{j}^{\text {eff }} / K\right) \rightarrow 1\right.$ and $\left.\sin \left(\pi x_{j}^{\mathrm{eff}} / K\right) \rightarrow \pi x_{j}^{\mathrm{eff}} / K \rightarrow 0\right)$ resulting in the mentioned simpler forms of Eqs. (11)-(13). These simpler equations provide us with the population inversion, $w_{j}$, induced dipole moment, $d_{j}$, and electric field of scattered light, $\epsilon_{j}$, at $2 N K$ discrete time points $\tau_{j}=j \pi / K(1 \leq j \leq 2 N K)$ equally separated by $\pi / K$. The required quantities $I_{j}$ are now to be found from the iterative Eq. (10) simplified by the replacement $\cot \left(\pi x_{j}^{\text {eff }} / 2 K\right) \rightarrow 2 K / \pi x_{j}^{\text {eff }}$. The advantage of the above simplifications is a considerable shortening of the calculation time of a personal computer used to find all $I_{j}, w_{j}, d_{j}$, and $\epsilon_{j}$. When calculating spectra of scattered light $(d(z), \epsilon(z))$ it is then recommended to apply the discrete Fourier transform to the list of the $d_{j}$ or/and $\epsilon_{j}$ values, instead of Eq. (14) or/and Eq. (15). In the above simpler version, the efficiency of our iterative method is competitive with the traditional approach based on the system of optical Bloch equations with no rotating-wave approximation. Typically, a few minutes is enough for a standard personal computer to get full information from our method, i.e., the dependencies $w(\tau), d(\tau), \epsilon(\tau), d(z)$, and $\epsilon(z)$, for a few-cycle incident pulse of arbitrary shape and strength from the field of extreme optics $(x \approx 1)$.

\section{Summary}

On the basis of a nonlinear Riccati-type equation, analytically solved in very short time intervals (shorter than half the optical period), we have formulated an effective iterative procedure for the problem of a two-level system exposed to a linearly polarized electromagnetic pulse. For different light-matter couplings (from weak through moderate to strong ones) we have proved very good quality of the procedure by recovering with it a number of subtle effects met in the previous numerically calculated photon-emission spectra and population inversion. Also, we have applied the iterative procedure to describe carrier-envelope phase effects in extreme nonlinear optics, particularly in population inversion and spectrum of coherently scattered light (in the regime of few-cycle pulses, these carrier-envelope phase effects are of current interest [24-29]). We would like to stress that no such detailed description of spectra of light scattered by two-level systems, as provided by our iterative method, is possible in the framework of the fully analytical but approximate (adiabatic) continuous-fraction theory of Plaja and Roso-Franco [30].

\section{References}

[1] L. Allen, J.H. Eberly, Optical Resonance and Two-Level Atoms, Wiley, New York 1975.

[2] R.W. Ziolkowski, J.M. Arnold, D.M. Gogny, Phys. Rev. A 52, 3082 (1995). 
[3] L.W. Casperson, Phys. Rev. A 57, 609 (1998).

[4] S. Hughes, Phys. Rev. Lett. 81, 3363 (1998).

[5] S. Hughes, Phys. Rev. A 62, 055401 (2000).

[6] V.P. Kalosha, J. Herrmann, Phys. Rev. Lett, 83, 544 (1999).

[7] A.V. Tarasishin, S.A. Magnitskii, A.M. Zheltikov, Opt. Commun. 193, 187 (2001).

[8] O.D. Mücke, T. Tritschler, M. Wegener, U. Morgner, F.X. Kärtner, Phys. Rev. Lett. 87, 057401 (2001).

[9] O.D. Mücke, T. Tritschler, M. Wegener, U. Morgner, F.X. Kärtner, Phys. Rev. Lett. 89, 127401 (2002).

[10] J. Xiao, Z. Wang, Z. Xu, Phys. Rev. A 65, 031402 (2002).

[11] J. Cheng, J. Zhou, Phys. Rev. A 67, 041404 (2003).

[12] T. Tritschler, O.D. Mücke, M. Wegener, U. Morgner, F.X. Kärtner, Phys. Rev. Lett. 90, 217404 (2003).

[13] B. Sundaram, P.W. Milonni, Phys. Rev. A 41, 6571 (1990).

[14] T. Zuo, S. Chelkowski, A.D. Bandrauk, Phys. Rev. A 48, 3837 (1993).

[15] T. Zuo, S. Chelkowski, A.D. Bandrauk, Phys. Rev. A 49, 3943 (1994).

[16] M.Yu. Ivanov, P.B. Corkum, Phys. Rev. A 48, 580 (1993).

[17] M.Yu. Ivanov, P.B. Corkum, P. Dietrich, Laser Phys. 3, 375 (1993).

[18] R. Bavli, H. Metiu, Phys. Rev. Lett. 69, 1986 (1992); Phys. Rev. A 48, 886 (1993); Phys. Rev. A 47, 3299 (1993).

[19] A. Levinson, M. Segev, G. Almogy, A. Yariv, Phys. Rev. A 49, 661 (1994).

[20] J.M.G. Llorente, J. Plata, Phys. Rev. A 45, 6958 (1992); Phys. Rev. E 49, 3547 (1994).

[21] G.M. Genkin, Phys. Rev. A 58, 758 (1998).

[22] R. Parzyński, M. Sobczak, Opt. Commun. 228, 111 (2003); J. Phys. B, At. Mol. Opt. Phys. 37, 743 (2004).

[23] T. Tritschler, O.D. Mücke, M. Wegener, Phys. Rev. A 68, 033404 (2003).

[24] U. Morgner, R. Ell, G. Metzler, T.R. Schibli, F.X. Kärtner, J.G. Fujimoto, H.A. Haus, E.P. Ippen, Phys. Rev. Lett. 86, 5462 (2001).

[25] D.B. Milošević, G.G. Paulus, W. Becker, Phys. Rev. Lett. 89, 153001 (2002).

[26] G.G. Paulus, F. Lindner, H. Walther, A. Baltuška, E. Goulielmakis, M. Lezius, F. Krausz, Phys. Rev. Lett. 91, 253004 (2003).

[27] A. Gürtler, F. Robicheaux, W.J. van der Zande, L.D. Noordam, Phys. Rev. Lett. 92, 033002 (2004).

[28] G. Sansone, C. Vozzi, S. Stagira, M. Pascolini, L. Poletto, P. Villoresi, G. Tondello, S. De Silvestri, M. Nisoli, Phys. Rev. Lett. 92, 113904 (2004).

[29] T. Brabec, F. Krausz, Rev. Mod. Phys. 72, 545 (2000).

[30] L. Plaja, L. Roso-Franco, J. Opt. Soc. Am. B 9, 2210 (1992). 